\title{
Astrophysical properties of binary star clusters in the Small Magellanic Cloud $\dagger$
}

\author{
João F. C. Santos Jr., ${ }^{1}$ Alex A. Schmidt ${ }^{2}$ and Eduardo Bica ${ }^{3}$ \\ ${ }^{1}$ Departamento de Física, ICEx, Universidade Federal de Minas Gerais, \\ Av. Antônio Carlos 6627, Belo Horizonte 31270-901, MG, Brazil \\ email: jsantos@fisica.ufmg.br \\ ${ }^{2}$ Departamento de Matemática, Universidade Federal de Santa Maria, Av. Roraima 1000, \\ Santa Maria 97105-900, RS, Brazil \\ ${ }^{3}$ Departamento de Astronomia, Universidade Federal do Rio Grande do Sul, \\ Av. Bento Gonçalves 9500, Porto Alegre 91501-970, RS, Brazil
}

\begin{abstract}
To study the evolution of binary star clusters, we have imaged seven systems in the Small Magellanic Cloud with the SOAR $4 \mathrm{~m}$ telescope using $B$ and $V$ filters. The sample contains pairs with well-separated components $(d<30 \mathrm{pc})$ as well as systems that apparently have merged, as evidenced by their unusual structures. By employing isochrone fitting to their color-magnitude diagrams, we have determined reddening values, ages and metallicities, and by fitting King models to their radial stellar-density profiles we estimated core radii. Disturbances of the density profiles are interpreted as evidence of interactions. Properties such as the distances between their components and their age differences are addressed in terms of the timescales involved, to assess the physical connection of the system. In two cases, the age difference is more than $50 \mathrm{Myr}$, which suggests a chance alignment, capture or sequential star formation.
\end{abstract}

Keywords. Magellanic Clouds, galaxies: star clusters

\section{Introduction}

Differently from the Galaxy, the low-density environment of the Magellanic Clouds favors the gravitational interaction of single clusters, which form binary (or multiple) groupings and will eventually merge or be disrupted. Pieces of evidence for these scenarios include: (i) many binary clusters in the Magellanic Clouds have been identified and recognized as true physically connected systems (Bhatia \& Hatzidimitriou 1988; Bhatia et al. 1991) and (ii) deep and precise colour-magnitude diagrams (CMDs) for isolated clusters in the Large Magellanic Cloud have revealed multiple population sequences (Mackey et al. 2008). If these facts are combined, their results indicate that we are witnessing different stages of cluster evolution in a context where cluster groupings and mergers are not uncommon. Evidence of cluster binarity and mergers has been reported in both Clouds, which are very rich in cluster pairs and multiples (Dieball et al. 2002; Bica et al. 2008; Carvalho et al. 2008). We determine astrophysical and structural parameters of SMC binary clusters by fitting isochrones to the CMDs and King functions to the radial-density profiles with the purpose of providing constraints on the evolution of such systems.

$\dagger$ The full poster (in pdf format) is available at http://www.astro.iag.usp.br/〜iaus266/Posters/pSantos.pdf. 
Table 1. Cluster sample and derived parameters.

\begin{tabular}{|c|c|c|c|c|c|c|c|c|c|}
\hline Cluster & $\begin{array}{c}\alpha 2000 \\
(\mathrm{~h}: \mathrm{m}: \mathrm{s})\end{array}$ & $\left(0^{\delta} 2000, \prime \prime\right)$ & $\begin{array}{c}E(B-V) \\
(\mathrm{mag})\end{array}$ & $\begin{array}{c}\text { age } \\
(\mathrm{Myr})\end{array}$ & $Z$ & $\begin{array}{l}R_{\mathrm{c}} \\
(\mathrm{pc})\end{array}$ & $\begin{array}{c}\Delta \text { age } \\
(\text { Myr })\end{array}$ & $\begin{array}{c}\text { separation } \\
(\mathrm{pc})\end{array}$ & $\begin{array}{c}\operatorname{age}(\text { lit.) } \\
(\text { Myr) }\end{array}$ \\
\hline NG C 220 & $0: 40: 31$ & $-73: 24: 10$ & $0.15 \pm 0.03$ & $70 \pm 10$ & 0.004 & $2.5 \pm 1.0$ & - & - & $\begin{array}{c}100 \pm 23^{a} \\
65 \pm 13^{b} \\
126^{c} \\
70-100^{d}\end{array}$ \\
\hline N G C 222 & $0: 40: 44$ & $-73: 23: 00$ & $0.15 \pm 0.03$ & $70 \pm 10$ & 0.004 & $1.5 \pm 0.5$ & 0 & 28 & $\begin{array}{c}100 \pm 23^{a} \\
70 \pm 7^{b} \\
100^{c} \\
70-100^{d}\end{array}$ \\
\hline NG C 241 & $0: 43: 33$ & $-73: 26: 25$ & $0.05 \pm 0.01$ & $80 \pm 10$ & 0.004 & $2.0 \pm 1.0$ & - & - & $\begin{array}{c}79 \pm 18^{a} \\
200^{c}\end{array}$ \\
\hline N G C 242 & $0: 43: 38$ & $-73: 26: 37$ & $0.05 \pm 0.01$ & $80 \pm 10$ & 0.004 & - & 0 & 9 & $79 \underset{63}{ }{ }^{c} 8^{a}$ \\
\hline B 78 & $0: 54: 45$ & $-72: 07: 46$ & $0.10 \pm 0.02$ & $45 \pm 5$ & 0.004 & $3.3 \pm 2.2$ & - & - & \\
\hline L 51 & $0: 54: 54$ & $-72: 06: 46$ & $0.10 \pm 0.02$ & $40 \pm 5$ & 0.004 & $1.5 \pm 0.3$ & 5 & 17 & \\
\hline IC 1611 & $0: 59: 48$ & $-72: 20: 02$ & $0.07 \pm 0.01$ & $140 \pm 30$ & 0.002 & $2.2 \pm 1.1$ & - & - & $\begin{array}{c}158 \pm 37^{a} \\
100 \pm 20^{b} \\
126^{c} \\
100 \pm 20^{e}\end{array}$ \\
\hline IC $1612 \mathrm{~W}=\mathrm{H} 86-186$ & $0: 59: 57$ & $-72: 22: 24$ & $0.07 \pm 0.01$ & $120 \pm 30$ & 0.002 & $2.5 \pm 0.5$ & - & - & $\begin{array}{c}180 \pm 20^{b} \\
126^{c}\end{array}$ \\
\hline IC $1612 \mathrm{E}=\mathrm{IC} 1612$ & $1: 00: 01$ & $-72: 22: 08$ & $0.07 \pm 0.01$ & $60 \pm 10$ & 0.002 & $2.0 \pm 1.0$ & 60 & 8 & $\begin{array}{c}50 \pm 24^{a} \\
100 \pm 50^{b} \\
100^{c}\end{array}$ \\
\hline NG C 376 & $1: 03: 53$ & $-72: 49: 34$ & $0.09 \pm 0.02$ & $50 \pm 10$ & 0.002 & $3.0 \pm 1.3$ & - & - & $\begin{array}{c}32 \pm 7^{a} \\
20 \pm 2 \\
16^{c} \\
25 \pm 10^{e}\end{array}$ \\
\hline K 50 & $1: 04: 36$ & $-72: 09: 38$ & $0.03 \pm 0.01$ & $50 \pm 10$ & 0.004 & $2.5 \pm 1.2$ & - & - & $20 \underset{8^{c}}{ \pm} 5^{a}$ \\
\hline $\begin{array}{l}\text { NG C } 422 \\
\text { IC } 1641\end{array}$ & $\begin{array}{l}1: 09: 25 \\
1: 09: 39\end{array}$ & $\begin{array}{l}-71: 46: 00 \\
-71: 46: 07\end{array}$ & $\begin{array}{l}0.06 \pm 0.01 \\
0.06 \pm 0.01\end{array}$ & $110 \pm 30$ & 0.004 & $1.4 \pm 0.5$ & - & - & \\
\hline IC 1641 & $1: 09: 39$ & $-71: 46: 07$ & $0.06 \pm 0.01$ & $500 \pm 30$ & 0.004 & $2.9 \pm 1.0$ & 390 & 20 & \\
\hline
\end{tabular}

${ }^{a}$ Pietrzynski \& Udalski (1999), ${ }^{b}$ de Oliveira et al. (2000), ${ }^{c}$ Chiosi et al. (2006), ${ }^{d}$ Matteucci et al. (2002), ${ }^{e}$ Piatti et al. (2007).

\section{Observations}

The SOAR optical imager (SOI) mounted in a bent-Cassegrain configuration on the $4.1 \mathrm{~m}$ SOAR telescope (Cerro Pachón, Chile) was employed to observe a sample of seven cluster merger candidates. Images in Bessel $B V$ filters were obtained on photometric nights (Oct/Nov 2007), using the SOI mini-mosaic of two E2V $2 \times 4 \mathrm{k}$ CCDs (1 pixel = $15 \mu \mathrm{m})$ to cover a $5.2 \times 5.2 \mathrm{arcmin}^{2}$ field of view. The CCDs were binned $2 \times 2$, yielding a scale of $0.154 \operatorname{arcsec}_{\text {pixel }}{ }^{-1}$. The average seeing was $\sim 0.95 \operatorname{arcsec}$ in $B$ and $\sim 0.8 \operatorname{arcsec}$ in $V$. Two images in each filter were obtained, with single exposure times of $480 \mathrm{~s}$ in $B$ and $195 \mathrm{~s}$ in $V$. The spatial scale is 1 arcsec $=0.3 \mathrm{pc}$. The CCD frames were reduced with IRAF software. STARFINDER (Diolaiti et al. 2000) was used to perform PSF photometry. Table 1 presents the cluster sample and includes the parameters derived here and from the literature. The $V$-band image of the interesting noncoeval pair IC 1641/NGC 422 is shown in Figure 1.

\section{Radial density profiles and structural parameters}

We built the radial density profile (RDP) for each merger candidate component by employing star counts in circular rings around the cluster center. For the sake of uniformity, all RDPs are based on rings $5^{\prime \prime}$ wide. To obtain an optimized RDP, we limit the data to a threshold magnitude for which the difference between the cluster central density and the adjacent field density is maximum. The cluster center is tweaked in the process. All stars fainter than this threshold are disregarded in the analysis. We employed two-parameter (central density $\sigma_{0}$, core radius $R_{\mathrm{c}}$ ) King profiles (King 1966) to derive 

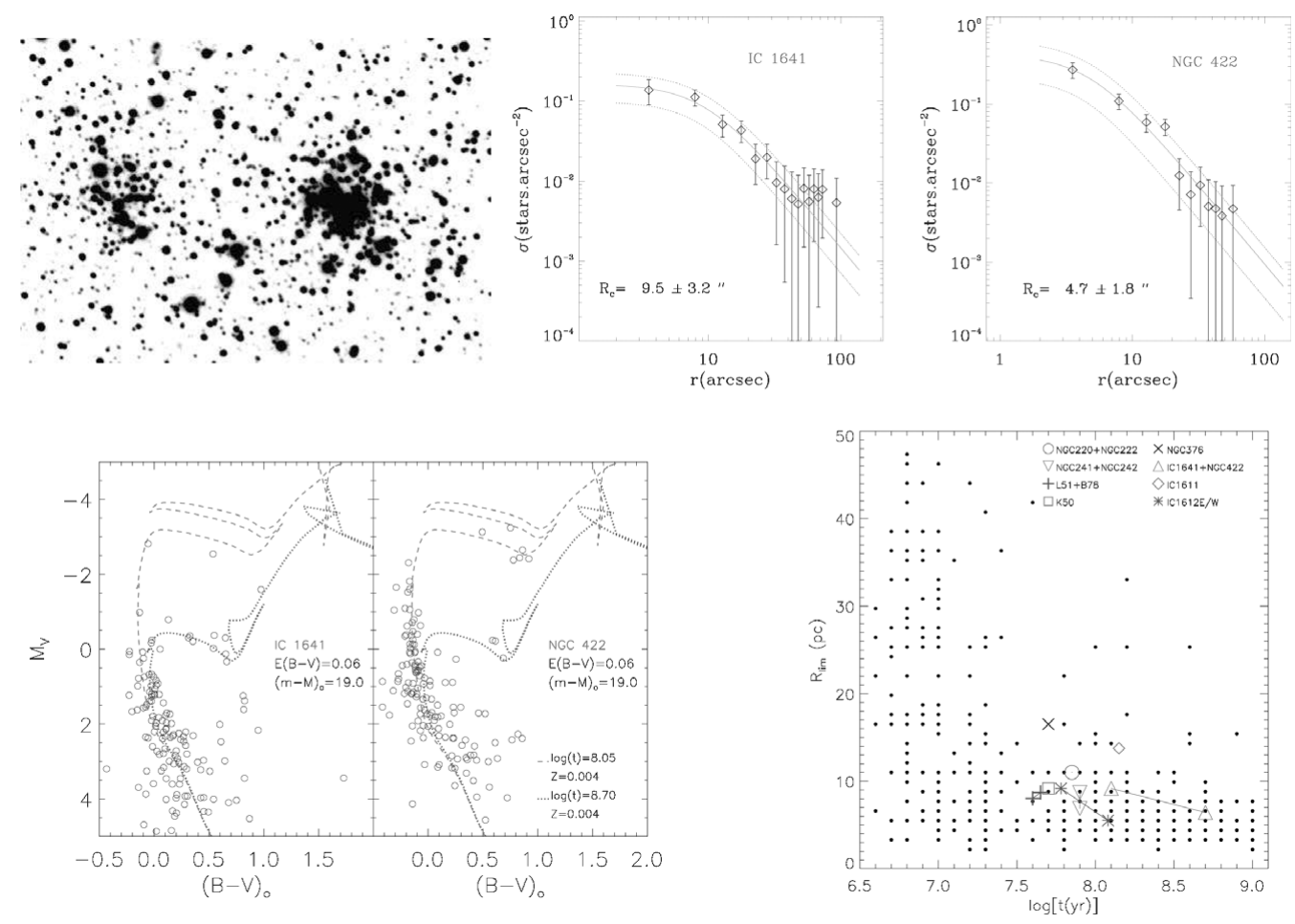

Figure 1. From top left to bottom right: (i) $V$ image extraction $\left(2.6^{\prime} \times 1.7^{\prime}\right)$ of pair IC 1641 (left) / NGC 422 (right); north is up and east to the left; (ii) The RDP on a logarithmic scale with a two-parameter King-profile fit superimposed, for which $R_{\mathrm{c}}$ is indicated; $1 \sigma$ dispersions of the fits are plotted as dotted lines; (iii) Isochrone fits to the CMDs of IC 1641 and NGC 422; for comparison, the best-matching isochrone for IC 1641 is also plotted in the CMD of NGC 422 and vice versa; (iv) Limiting radius (Bica et al. 2008) as a function of age; the dots represent SMC clusters from Chiosi et al. (2006) and other symbols identify the present sample of binary/merger clusters.

structural properties. The background was determined by fitting a constant to the outermost four rings, which was then subtracted from the overall stellar density before the King profile fitting was performed. Figure 1 shows the RDP for clusters IC 1641/NGC 422.

\section{CMDs and astrophysical parameters}

We obtained astrophysical parameters by fitting Padova isochrones (Girardi et al. $2002)$ to the $V$ versus $(B-V)$ CMDs. To minimize contamination of background stellar fields, a circular region defined by a limiting radius $\left(R_{\text {lim }}\right)$ covering the cluster central area was selected. $R_{\text {lim }}$ is defined as the radius where the cluster stellar density begins to stand out from the background. For all clusters in the sample, good fits were obtained for $(m-M)_{0}=19.0 \pm 0.1 \mathrm{mag}$. Reddening, age and metallicity corresponding to the best-matching isochrones are presented in Table 1 . Figure 1 shows isochrone fits to the observed CMDs of IC 1641 and NGC 422.

\section{Discussion}

In general, our age estimates agree with CMD-based age determinations in the literature (see Table 1). The ages of L51/B78 and NGC422/IC1641 have been determined 
using isochrone fits for the first time. Because of a disturbed surface-brightness profile and isophotal features, Carvalho et al. (2008) classified K50 and NGC 376 as mergers and the pairs IC 1612E/W and NGC 241/242 as true binary clusters. Except for NGC 422/IC 1641 and IC 1612E/W, all cluster pairs in our sample are nearly coeval and have ages close to $10^{7} \mathrm{yr}$, consistent with the timescales of the dynamical models. NGC 422/IC 1641 and IC 1612E/W have ages that differ by $390 \mathrm{Myr}$ and $60 \mathrm{Myr}$ respectively, implying that, if they are interacting, a capture process may have occurred. Another possibility is that the oldest cluster in the pair triggered the star formation that led to the youngest through supernova explosions and shell expansion. Such a mechanism has also been proposed as a plausible explanation by Vallenari et al. (1998) for the LMC pair NGC 1903/SL 357, although their age difference is $550 \mathrm{Myr}$.

As regards the timescales involved, the expected effect of the gravitational interaction for clusters with a mass of $10^{5} \mathrm{M}_{\odot}$ and a half-mass radius of $10 \mathrm{pc}$, is that they will merge in $10^{7} \mathrm{yr}$ if their separation is $\sim 25 \mathrm{pc}$ (Sugimoto \& Makino 1989). Models for less massive clusters in the LMC tidal field predict a few $\times 10^{7} \mathrm{yr}$ for the pair lifetime, with separations varying from $\sim 6 \mathrm{pc}$ for cluster masses of $10^{3} \mathrm{M}_{\odot}$ and $\sim 14 \mathrm{pc}$ for $10^{4} \mathrm{M}_{\odot}$ (Bhatia 1990). According to these models, after this timescale either the cluster pair merges due to mutual gravitational interaction or is disrupted by the galactic tidal field and, less probably, by passing giant molecular clouds.

Clusters resulting from pairs that have merged are expected to show structural properties which differ from those of single clusters. The plot of $R_{\text {lim }}$ versus age indicates that K50, NGC 376 and IC 1611 have a larger $R_{\text {lim }}$ than the mean locus of single clusters, which may be additional evidence of their merger status (Mackey \& Gilmore 2003).

\section{Acknowledgements}

We thank the Brazilian agencies CNPq and FAPEMIG for financial support.

\section{References}

Bhatia, R. K. 1990, PASJ, 42, 757

Bhatia, R. K. \& Hatzidimitriou, D. 1988, A\&A, 230, 215

Bhatia, R. H., Read, M. A., Tritton, S., \& Hatzidimitriou, D. 1991, A\& AS, 87, 335

Bica, E., Bonatto, C., Dutra, C. M., \& Santos Jr., J. F. C. 2008, MNRAS, 389, 678

Carvalho, L., Saurin, T. A., Bica, E., Bonatto, C., \& Schmidt, A. A. 2008, A\& A, 485, 71

Chiosi, E., Vallenari, A., Held, E. V., Rizzi, L., \& Moretti, A. 2006, A\&A, 452, 179

de Oliveira, M. R., Dutra, C. M., Bica, E., \& Dottori, H. 2000, A\&AS, 146, 57

Dieball, A., Müller, H., \& Grebel, E. K. 2002, A\& $A$, 391, 547

Diolaiti, E., Bendinelli, O., Bonaccini, D., Close, L., Currie, D., \& Parmeggiani, G. 2000, A\&SAS, 147,335

Girardi, L., Bertelli, G., Bressan, A., Chiosi, C., Groenewegen, M. A. T., Marigo, P., Salasnich, B., \& Weiss, A. 2002, A\& A, 391, 195

King, I. 1966, AJ, 71, 64

Mackey, A. D., Broby Nielsen, P., Ferguson, A. M. N., \& Richardson, J. C. 2008, ApJ (Letters), 681, L17

Mackey, A. D. \& Gilmore, G. F. 2003, MNRAS, 338, 120

Matteucci, A., Ripepi, V., Brocato, E., \& Castellani, V. 2002, A\&SA, 387, 861

Piatti, A. E., Sarajedini, A., Geisler, D., Clark, D., \& Seguel, J. 2007, MNRAS, 377, 300

Pietrzyński, G. \& Udalski, A. 1999, AcA, 49, 157

Sugimoto, D. \& Makino, J. 1989, PASJ, 41, 1117

Vallenari, A., Bettoni, D., \& Chiosi, C. 1998, A\&SA, 331, 506 